\title{
HAPLOTYPES OF FOXP3 GENETIC VARIANTS ARE ASSOCIATED WITH SUSCEPTIBILITY, AUTOANTIBODIES, AND TGF- $\beta 1$ IN PATIENTS WITH SYSTEMIC LUPUS ERYTHEMATOSUS
}

Nicole Perugini Stadtlober ${ }^{1}$, Tamires Flauzino ${ }^{1}$, Lorena Flor da Rosa Franchi Santos ${ }^{1}$, Tatiana Mayumi Veiga Iriyoda ${ }^{2, *}$, Neide Tomimura Costa ${ }^{1}$, Marcell Alysson Batisti Lozovoy ${ }^{1}$, Isaias Dichi ${ }^{1}$, Edna Maria Vissoci Reiche ${ }^{1}$, Andréa Name Colado Simão

1.Universidade Estadual de Londrina, Londrina (PR), Brazil; 2.Pontifícia Universidade Católica do Paraná, Londrina (PR), Brazil.

*Corresponding author: tatimayumi54@gmail.com

\section{BACKGROUND}

Systemic lupus erythematosus (SLE) is an autoimmune inflammatory disease, characterized by dysregulated innate and adaptive immune responses. The regulatory $\mathrm{CD} 4+\mathrm{CD} 25+\mathrm{T}$ (Treg) cells plays a crucial role in suppression of the immune response. The FoxP3 belongs to the forkhead/winged-helix family of transcription factors and it is considered the master regulator of Treg cells development and function. Different single nucleotide variants (SNVs) have been described in the promoter region of FOXP3, which can affect the expression of FOXP3 and impair the Treg cells differentiation and function. The -924 G>A (rs2232365) and -3279 C>A (rs3761548) FOXP3 variant were associated with the susceptibility and prognosis of SLE. Thus, the aim of this study was to evaluate the association of rs2232365 (-924 G>A) and rs3761548 (-3279 C>A) FOXP3 variants with SLE susceptibility, TGF- $\beta 1$ plasma levels, autoantibodies, lupus nephritis (LN), and SLE disease activity index (SLEDAI).

\section{METHODS}

The study included 196 SLE female patients and 157 female controls. FOXP3 variants were determined with polymerase chain reaction-restriction fragment length polymorphism (PCR-RFLP). Plasma levels of TGF- 1 were determined using immunofluorimetric assay.

\section{RESULTS}

Based on results, the median of C3 was $112.5 \mathrm{mg} / \mathrm{dL}$ (92-133) and C4 $20.1 \mathrm{mg} / \mathrm{dL}$ (13.5-25.9). The median of antinucleosome levels was $53.74 \mathrm{IU} / \mathrm{mL}$ (19.07-138.74), 58.2\% had positive anti-dsDNA antibodies, $23.1 \%$ had positive anti-SM antibodies and $43.2 \%$ had positive anti-U1RNP antibodies. In addition, 87 (46.5\%) patients had LN. The AA genotype (OR $=2.650, \mathrm{Cl} 95 \%=1.070-6.564$, $\mathrm{p}=0.035)$ and $\mathrm{A}$ allele $(\mathrm{OR}=2.644, \mathrm{Cl} 95 \%=1.104-6.333, \mathrm{p}=0.029)$ were associated with SLE diagnosis in the $-3279 \mathrm{C}>\mathrm{A}$. The A/A haplotype was associated with SLE (OR =3.729, CI 95\% =1.006-13.820, $p=0.049)$. GCGC haplotype patients had higher TGF- $\beta 1$ levels $(p=0.012$ ) than other haplotypes. Patients with -924 AA genotype showed higher frequency of anti-dsDNA $(p=0.012)$ and anti-U1RNP $(p=0.036)$. The A/C haplotype had higher SLEDAI score $(O R=1.119, C l 95 \%=1.015-1.234), p=0.024)$ and ACAC haplotype higher frequency of anti-dsDNA ( $O R=3.026, \mathrm{Cl} 95 \%=1.062-8.624, p=0.038)$, anti- U1RNP (OR $=5.649$, $\mathrm{Cl} 95 \%=1.199-26.610, \mathrm{p}=0.029]$ and nephritis $(\mathrm{OR}=2.501, \mathrm{Cl} 95 \%=1.004-6.229, \mathrm{p}=0.049)$.

\section{CONCLUSION}

Our data demonstrate that the $\mathrm{G} / \mathrm{C}$ haplotype provides protection for SLE. While the presence of allele A of both variants could favor autoimmunity, disease activity, and LN.

\section{KEYWORDS}

Systemic lupus erythematosus, TGF- $\beta 1$, FOXP3, rs2232365, rs3761548. 